\title{
Targeted Muscle Reinnervation and Advanced Prosthetic Arms
}

\author{
Jennifer E. Cheesborough, MD ${ }^{1}$ Lauren H. Smith, BS 2,3 Todd A. Kuiken, MD 2,3,4 \\ Gregory A. Dumanian, MD ${ }^{1}$
}

${ }^{1}$ Division of Plastic and Reconstructive Surgery, Northwestern

University, Chicago, Illinois

${ }^{2}$ Center for Bionic Medicine, Rehabilitation Institute of Chicago,

Chicago, Illinois

${ }^{3}$ Department of Biomedical Engineering, Northwestern University, Chicago, Illinois

${ }^{4}$ Department of Physical Medicine and Rehabilitation, Northwestern

University, Chicago, Illinois

Semin Plast Surg 2015;29:62-72.

\begin{abstract}
Address for correspondence Gregory A. Dumanian, MD, Division of Plastic and Reconstructive Surgery, Northwestern University, 675 N. St. Clair, Suite 19-250, Chicago, IL 60610, (e-mail: gdumania@nmh.org).
\end{abstract}

\author{
Abstract \\ Keywords \\ - targeted muscle \\ reinnervation \\ - amputation \\ - prosthetic limb \\ - transhumeral \\ amputation \\ - shoulder \\ disarticulation
}

Targeted muscle reinnervation (TMR) is a surgical procedure used to improve the control of upper limb prostheses. Residual nerves from the amputated limb are transferred to reinnervate new muscle targets that have otherwise lost their function. These reinnervated muscles then serve as biological amplifiers of the amputated nerve motor signals, allowing for more intuitive control of advanced prosthetic arms. Here the authors provide a review of surgical techniques for TMR in patients with either transhumeral or shoulder disarticulation amputations. They also discuss how TMR may act synergistically with recent advances in prosthetic arm technologies to improve prosthesis controllability. Discussion of TMR and prosthesis control is presented in the context of a 41-year-old man with a left-side shoulder disarticulation and a right-side transhumeral amputation. This patient underwent bilateral TMR surgery and was fit with advanced pattern-recognition myoelectric prostheses.
In the United States in 2005, there were 1.7 million people living with limb amputations; that number is expected to double by $2050 .{ }^{1}$ In addition to the impact on civilians, limb loss affects a significant portion of our servicemen and women. From 2001 to 2010, over 1,000 U.S. military personnel suffered traumatic major limb amputations in the Iraq and Afghanistan conflicts. ${ }^{2}$ Furthermore, many of our wounded warriors are returning with multiple limb amputations, resulting in a much greater impairment.

Achieving a high level of function with prosthetic limbs remains challenging, especially for upper extremity amputations at the elbow or higher, where the disability is greatest. Motorized hooks, hands, wrists, and elbows are commercially available, but precise control is lacking. Currently, most motorized artificial limbs are controlled with the surface electromyogram (EMG) from a residual pair of agonist-antagonist muscles in the amputated limb. ${ }^{3}$ This method allows for isolated motion, but not coordinated motion such as elbow flexion and handgrip. Furthermore, traditional methods of myoelectric control are awkward to learn, as they do not employ native cortical signals to direct fluid motion.

Targeted muscle reinnervation (TMR) is a surgical nervetransfer procedure, developed to provide amputees with more intuitive control of upper limb prostheses by combining available technology with modification of the residual limb anatomy. Residual nerves from the amputated limb are transferred to new muscle targets that have otherwise lost their function. As part of the nerve transfer, the target muscles are separated from their native motor nerve input so that the newly transferred nerve can reinnervate them. These reinnervated muscles then serve as biological amplifiers of the amputated nerve motor signals. By transferring multiple nerves, TMR myoelectric signals allow intuitive, simultaneous control of multiple joints in an advanced prosthesis.
Issue Theme Complex Upper Extremity Reconstruction; Guest Editors, Morad Askari, MD, and Steven L. Moran, MD
Copyright @ 2015 by Thieme Medical Publishers, Inc., 333 Seventh Avenue, New York, NY 10001, USA. Tel: +1(212) 584-4662.
DOI http://dx.doi.org/ 10.1055/s-0035-1544166. ISSN 1535-2188. 
Clinical observations by many surgeons have indicated that TMR also yields significant improvement in neuroma pain. ${ }^{4}$ Approximately $25 \%$ of major limb amputees will develop chronic localized pain due to symptomatic neuromas in the residual limb. ${ }^{5-7}$ Neuromas consist of disorganized axons encased in scar and form at the proximal end of a severed or damaged nerve. Neuromas are a result of uncoordinated attempts of nerve fibers to regenerate, and can cause focal pain that is often difficult to treat medically or surgically. Neuromas are responsible for much of the residual limb pain experienced after a traumatic amputation. In addition, neuromas frequently make wear and use of prosthesis uncomfortable or even impossible, thus reducing the functional ability of the individual. Over 150 surgical treatments for end-neuromas have been described in the literature ${ }^{8}$; however, this myriad of treatments only highlights the fact that no single neuroma treatment has been shown to consistently work well or better than the others. Compared with other treatments, TMR-style nerve transfers provide an "end organ" for the nerve to innervate; in laboratory animals, this has been shown to return the neuroma to a much more normal architecture. In a novel rabbit amputation-neuroma model, nerve transfers between an amputated forelimb nerve stump with a neuroma and a pedicled rectus abdominis muscle motor nerve yielded a nerve morphology more similar to uninjured, normal nerve than the excised neuroma. $^{9-11}$

In TMR, after excision of the neuroma, the residual nerve stumps are coapted to cut motor nerves that innervate new target muscles. Targeted muscle reinnervation provides a physiologically appropriate environment for regenerating axons, encouraging organized nerve regeneration into target muscles, and preventing the chaotic and misdirected nerve growth that leads to neuroma formation. Early clinical results demonstrate significant improvement in neuroma pain after TMR in upper extremity amputees. ${ }^{4}$ Targeted muscle reinnervation gives the nerves somewhere to go and something to do-elements lacking in other neuroma treatments.

\section{TMR Techniques}

\section{Elbow Disarticulation and Transhumeral Amputation}

An elbow disarticulation residual limb is a challenging level of amputation. When not wearing a prosthesis, the residual limb is long enough to reach a table easily and is generally more functional than a higher-level transhumeral amputation. However, wearing a prosthesis has unique challenges for elbow disarticulation amputees. For body-powered devices, external hinges can be used, but the prosthesis is bulky at the elbow and the hinges frequently catch on clothing. It is nearly impossible for an elbow disarticulation amputee to wear prosthesis with a motorized elbow because current myoelectric elbows add 5 to $6 \mathrm{~cm}$ of length to the upper arm section. This same problem exists for long transhumeral amputees.

For patients with elbow disarticulations who are motivated to have better limb length symmetry while wearing a prosthesis or to be able to wear a motorized elbow, there are several surgical options. First, the limb can be simply shortened to allow for the necessary prosthetic componentry. Another approach would be to perform a shortening humeral osteotomy, removing $\sim 5$ to $6 \mathrm{~cm}$ of midshaft bone. Although this is a more technically demanding procedure, it preserves the humeral condyles, which are useful for prosthetic suspension and can enable the patient to perform some humeral rotation.

With long transhumeral amputations, a standard shortening of the humerus can be performed to allow enough room for the prosthetic componentry. Alternatively, an angulation osteotomy of the distal humerus will provide a lever arm for prosthesis suspension and additional rotational control. ${ }^{12,13}$ As opposed to the Marquardt osteotomy, ${ }^{14}$ we recommend a longer distal bone segment of 6 to $8 \mathrm{~cm}$, an angle of 70 degrees, and a posterior fixation plate that will support the new bone shape over time (-Fig. 1 ).

After myodesis and assurance of adequate soft tissue coverage of the distal bone, the nerve endings are addressed individually. Targeted muscle reinnervation should be considered at the time of elective amputation (or in the revision process after trauma, when the necessary incisions and soft tissue manipulation are safe to perform). This can avoid additional surgery, allow for nerve transfer when the nerves are unscarred and at their greatest length, and speed up the patient's rehabilitation process enabling him or her to learn how to use a myoelectric prosthesis with a full complement of control signals at the earliest stage. ${ }^{12,15}$ The nerve transfers associated with TMR are designed to recapture the motor
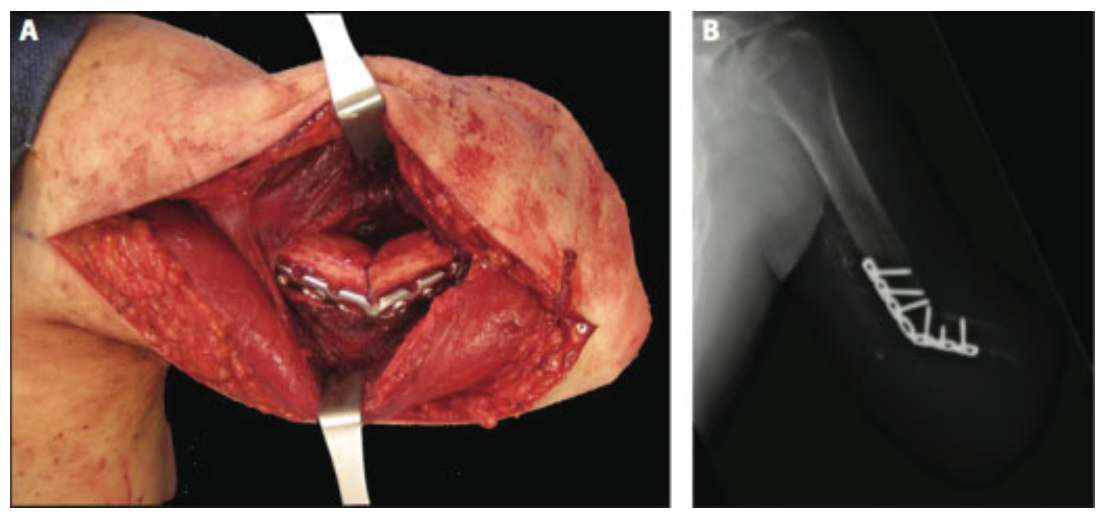

Fig. 1 (A) Posterolateral view of angulation osteotomy in a transhumeral amputee. (B) X-ray of angulation osteotomy. 
control information still retained in the central and peripheral nervous systems that has become inaccessible due to loss of muscle effectors as a result of the amputation. After transfer, the major upper extremity nerves will cause contraction of their new target muscles and the EMG signals will be sensed by the prosthesis allowing for coordinated, intuitive function. It is important to note that cut sensory and mixed nerves can form symptomatic neuromas, but that cut motor nerves do not.

There are two separate incisions made in the upper arm, one ventral and one dorsal. A nerve stimulator is extremely helpful in localizing and identifying individual motor nerves. Through the longitudinal ventral incision, centered over the muscular raphe, the median nerve is transferred end-to-end to the motor nerve of the short head of the biceps to provide a "hand close" signal (-Fig. 2). The long head of the biceps, innervated by the musculocutaneous nerve, is left intact to maintain the "elbow flexion" signal. Through the dorsal longitudinal incision, the long head of the triceps is left intact to provide an "elbow extension" signal, while the distal radial nerve is transferred to the motor nerve of the lateral head of
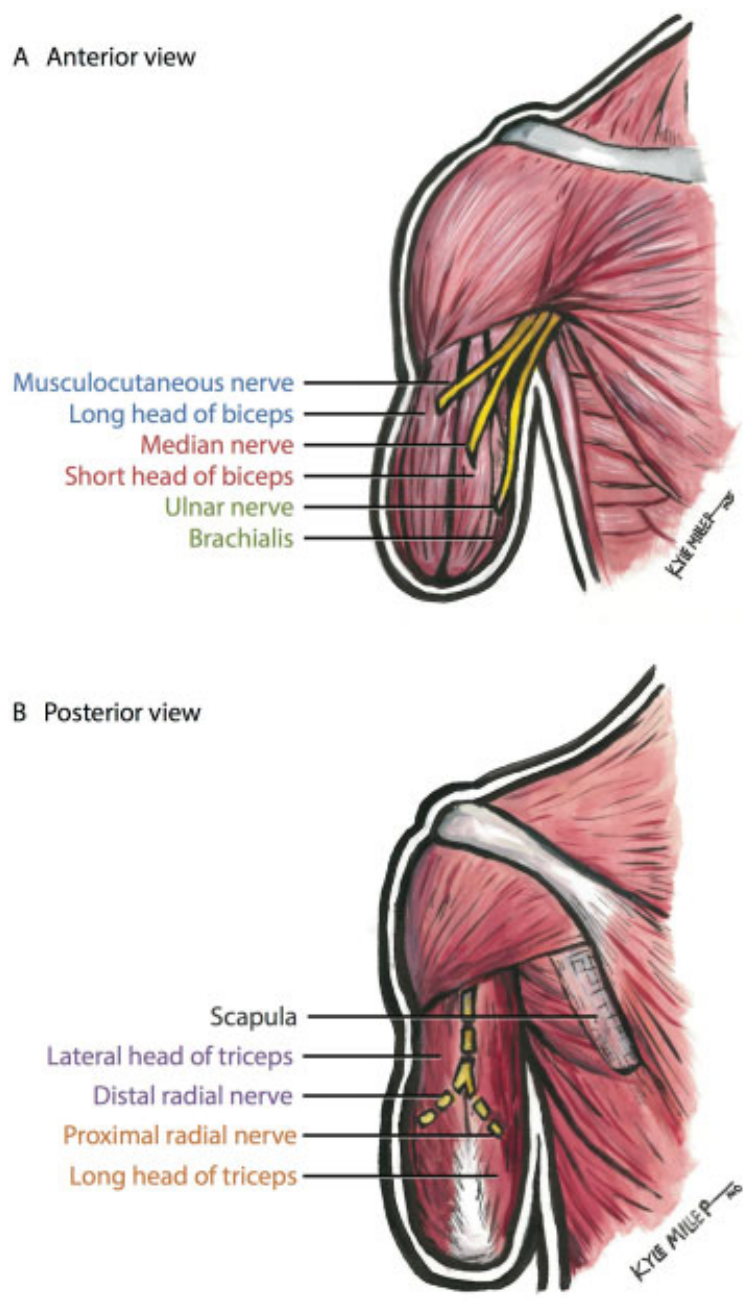

Fig. 2 Schematic of typical surgical plan for transhumeral targeted muscle reinnervation. (A) Anterior view. (B) Posterior view. Colormatched labels represent muscles and their source of innervation. the triceps to provide a "hand open" signal. If adequate limb length remains and the brachialis muscle is present, the ulnar nerve is transferred to the motor nerve entering the brachialis muscle to provide an additional hand or wrist control signal. It is advantageous for later signal acquisition to raise an adipofascial flap at the time of initial incision, and to place this tissue between the muscle bellies of the biceps or triceps to separate the future myoelectric signals.

\section{Shoulder Disarticulation}

Targeted muscle reinnervation at the shoulder disarticulation level is more challenging than transhumeral amputations due to shorter donor nerve length, fewer muscle targets, and the frequently encountered soft tissue distortion. The goals for hand open/close and elbow flexion/extension signals remain the same. Target muscles include the pectoralis major, pectoralis minor, serratus anterior, and latissimus dorsi muscles. The pectoralis major muscle may be further subdivided into the clavicular head, the cranial sternal head, and the caudal sternal head, depending on its innervation pattern.

The most common nerve transfer pattern involves four coaptations (- Fig. 3). ${ }^{12,15,16}$ The musculocutaneous nerve is the highest priority for transfer as it alone can be used for elbow control if needed. It is transferred to the clavicular head of the pectoralis major to achieve an elbow flexion signal. The clavicular head of the pectoralis major gives particularly good EMG signals due in part to its close proximity to the clavicular bone. ${ }^{17}$ The median nerve is the second priority and is transferred to the largest motor nerve of the sternal head of the pectoralis muscle to provide a hand close signal. Close attention should be paid to the radial nerve as a proximal branch may be connected to residual triceps muscle and this native innervation pattern would allow for an elbow extension signal without nerve transfer. The distal radial nerve can be transferred to the latissimus dorsi (dividing the thoracodorsal nerve) or serratus anterior (dividing the long thoracic nerve) to provide a hand open signal. Alternatively, if the motor nerve to the pectoralis minor can be reached by the residual radial nerve, this muscle can be disinserted and mobilized laterally to provide a target for the radial nerve distinct from the pectoralis major. The ulnar nerve is the

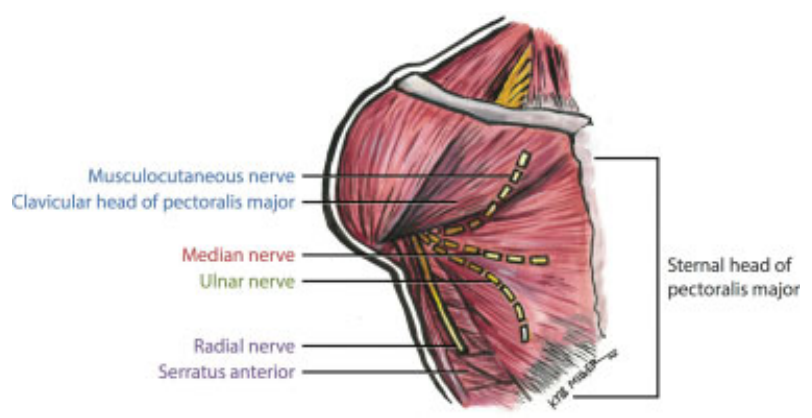

Fig. 3 Schematic of typical surgical plan for shoulder disarticulation targeted muscle reinnervation. Color-matched labels represent muscles and their source of innervation. In this example, the sternal head of pectoralis major is partitioned into two parts and reinnervated by the median and ulnar nerves. 
lowest priority nerve because it has both hand open and hand close signals when activated. However, it is clearly worth transferring if a suitable target muscle is available. It may be transferred to any remaining muscular targets for an additional hand or wrist signal. None of these transfers is a hard and fast rule, but rather a suggested pattern, one that allows for improvisation and creativity in the face of challenging traumatically injured residual limbs.

\section{Management of Complex Amputations}

Patients with amputations due to trauma or electrical injury often have extensive soft tissue damage, which compromises the ability to wear prostheses comfortably. Scars, friable skin grafts, bony prominences, and contour irregularities prevent consistent socket suction and fit. Skin breakdown is common and leads to pain, wound infections, and interruptions in prosthetic wear. Although the prosthetist can often overcome these challenges with prosthesis modification, consultation with the plastic surgeon may lead to a more permanent improvement in the soft tissue.

Many options exist for soft tissue improvement. Local tissue rearrangement may include rotation flaps, advancement flaps, or Z-plasty for release of scar contractures. In the process of exposing the nerves and target muscles of the upper extremity or chest for TMR, proximally based adipofascial flaps are elevated. This serves two important purposes: (1) It allows for thinning of the subcutaneous adipose tissue, which amplifies the EMG signal sensed on the skin; and (2) it allows for placement of these flaps between target muscle segments to help isolate their individual signals. Patients with excessive subcutaneous fat that dampens the EMG signal and impedes socket fit may undergo direct lipectomy or circumferential liposuction to reduce the overall thickness of the subcutaneous tissue.

Heterotopic ossification (traumatic myositis ossificans) remains a problem for many amputees. This ectopic bone within the soft tissue is often painful and causes breakdown of the overlying soft tissues especially in areas of prosthesis wear. Although the exact etiology of heterotopic ossification (HO) is largely unknown, it is thought that the trauma and subsequent inflammation of the amputation limb leads to transformation of primitive mesenchymal cells into osteogenic cells. ${ }^{18}$ Treatment includes physical therapy, diphosphonates, and nonsteroidal anti-inflammatory drugs, radiation, and surgical excision of mature HO. ${ }^{19}$ Historically, it has been recommended to wait 6 to 12 months for the $\mathrm{HO}$ to mature before surgical excision. ${ }^{20}$ We disagree and believe HO should be removed when it becomes problematic enough to impede the use of the prosthesis or causes other significant problems for the patient. When planning for excision, the surgeon should ensure there is adequate soft tissue coverage of the excisional site to prevent delayed healing and prolonged inflammation, factors that contribute to recurrence of HO.

Skin grafts are an excellent means to close a wound, especially a large traumatic wound. However, they are prone to breakdown when subjected to daily prosthesis wear, especially over bony prominences. Placement of a tissue expander beneath adjacent, unaffected skin may allow for excision of the skin graft and replacement with supple, durable skin and subcutaneous tissue. Alternatively, local pedicled flaps or free tissue transfer may be employed to improve the soft tissue envelope. When available, the latissimus dorsi myocutaneous flap is a workhorse flap for resurfacing of the transhumeral and shoulder disarticulation amputee residual limbs.

Free tissue transfer should be considered for soft tissue improvement, but also for the addition of muscular targets when no other local targets for TMR are available. In patients with a dearth of muscular targets at the shoulder disarticulation level, a free serratus anterior muscle flap can be transferred with each slip serving as a separate target for reinnervation by the brachial plexus donor nerves. In transhumeral amputees, the gracilis muscle or the rectus abdominis muscle may be transferred to replace missing biceps or triceps muscles as both targets for reinnervation and bulk to support a prosthesis. Both of these muscles have segmental innervation allowing for multiple targets for reinnervation.

\section{Case Example}

A 41-year-old man presented in 2011 following a highvoltage injury sustained 1 year prior while at work as a lineman. He had suffered extensive burns requiring amputation of both arms, the left at the shoulder and the right at the transhumeral level. Closure of his wounds had required extensive split thickness skin grafting. He had significant residual limb pain, chronic wound breakdown, and poor prosthesis control partly due to his inability to wear a prosthesis consistently because of skin breakdown and pain. Certain challenges were apparent from the start (-Fig. 4). This man had inadequate soft tissue to suspend a prosthesis on his right residual limb. He had thin skin grafts overlying bony prominences. In particular, the left scapula had extensive $\mathrm{HO}$ and no soft tissue coverage. It caused significant pain such that he could not lie on his back to sleep and the thin skin kept breaking down with wear of the left prosthesis. He had significant neuroma pain that frequently prevented him from donning his prosthesis at all. When he could wear the prostheses, he had very limited function.

A staged approach was planned to address the soft tissue envelope and the nerve transfers of the bilateral upper extremities. The left chest wall (shoulder disarticulation) was addressed first as this site caused the most significant pain and was troubled most by chronic wounds (-Fig. 5). Two large 600-cc tissue expanders were placed beneath the unaffected anterior chest skin and subcutaneous tissue to facilitate excision of the skin graft directly overlying the acromion (bony prominence) and the lateral chest wall.

After 4 months, the patient underwent simultaneous posterior excision of $\mathrm{HO}$ over the scapula with soft tissue coverage and anterior targeted muscle reinnervation. The expanded skin was advanced to cover the lateral chest and acromion. At the same time, a pedicled myocutaneous latissimus flap was rotated superiorly and laterally to cover the scapula. Prior to closure of the skin envelope, all nerve 

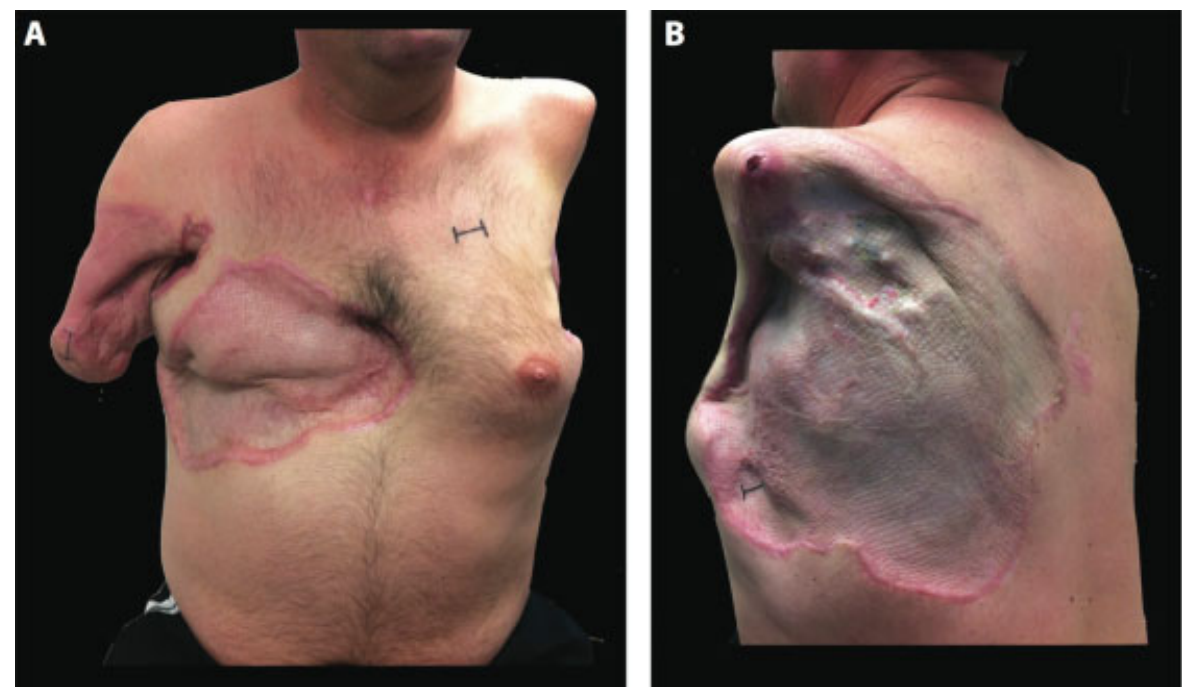

Fig. 4 The case report patient with left shoulder disarticulation and right transhumeral amputation with loss of significant volar musculature. (A) Anteroposterior view. (B) Oblique view.
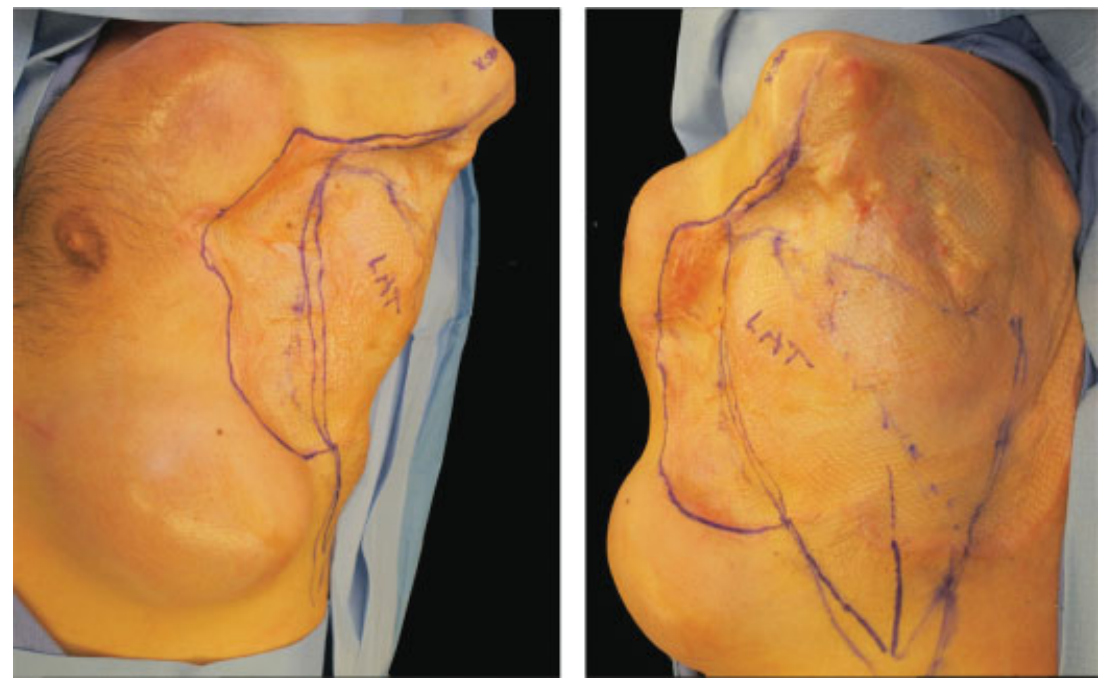

Fig. 5 Left chest with tissue expanders in place and planned latissimus rotation flap. Oblique views.

transfers were performed (-Fig. 6). The musculocutaneous nerve was transferred to the motor nerve to the clavicular head of pectoralis major. The median nerve was split along a natural cleavage plane and transferred to the medial and lateral branches of the sternal head of the pectoralis major. The radial nerve was transferred to the motor branch of the long thoracic nerve to the serratus anterior. Finally, the pectoralis minor muscle was disinserted and rotated laterally so that it no longer was underneath the pectoralis major muscle and the ulnar nerve was transferred to the motor nerve entering the pectoralis minor. The skin envelope was closed and all but a small patch of skin graft could be excised. Four months later, the left-sided soft tissues had healed well, his pain was significantly improved, and the patient was ready for surgery to the right residual limb.
The challenges specific to the right, transhumeral limb included a paucity of soft tissue to allow for socket suspension, an absent biceps muscle limiting the number of target muscles, and pain related to multiple neuromas ( - Fig. 7 ). To address these issues with one combined procedure, the patient underwent a free myocutaneous gracilis flap to the anterior right humerus and targeted muscle reinnervation. The specific nerve transfers were transfer of the musculocutaneous nerve to the proximal motor nerve to gracilis, transfer of the median nerve to the distal motor nerve to gracilis, and transfer of the radial nerve to the motor branch of the lateral triceps. Due to a dearth of targets, the ulnar nerve neuroma was excised and buried within the deltoid muscle. The free tissue transfer of the gracilis muscle provided two new nerve targets due to its segmental innervation and 


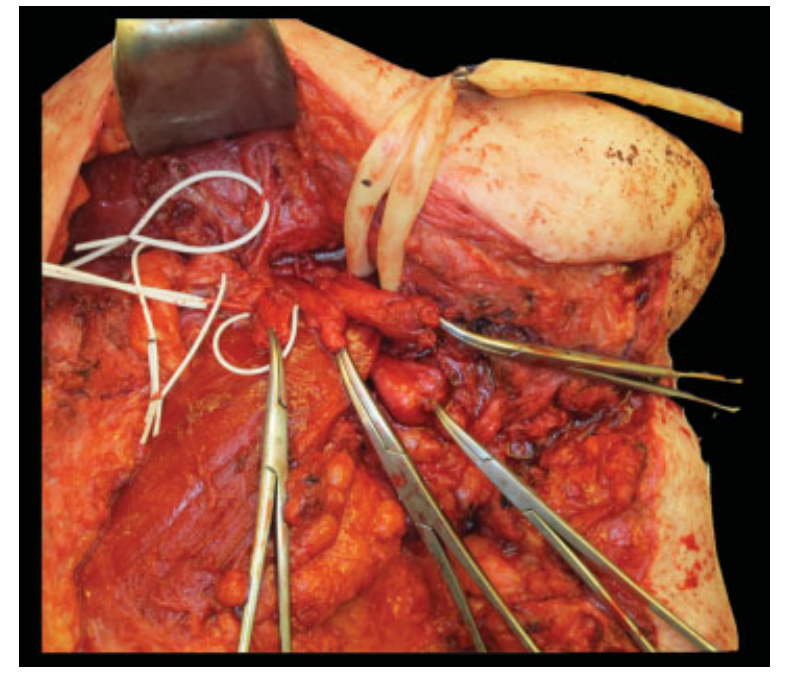

Fig. 6 Identification of ulnar, median, musculocutaneous, and radial nerves during left shoulder disarticulation targeted muscle reinnervation.

provided much needed bulk to the residual limb to improve socket comfort and suspension. A split thickness skin graft was applied to the gracilis muscle to close the wound and allow for maximal transcutaneous signal reception by the future prosthesis. A thin skin graft over muscle is desirable for signal interpretation; however, a thin skin graft over a bony prominence is detrimental due to the tendency to develop wound breakdown from pressure.

Five months following the completion of these three staged procedures, the prosthetists were able to detect multiple independent TMR-related signals on both upper extremities. Anecdotally, the patient also noted complete resolution of his neuroma pain bilaterally. This is a phenomenon that we have seen in over $94 \%$ of the upper extremity TMR patients at our institution. ${ }^{4}$ The patient still has occasional phantom limb pains, but he does not have any more chronic localized pain. With a stable soft tissue envelope to enable prosthesis wear (-Fig. 8) and multiple independent,

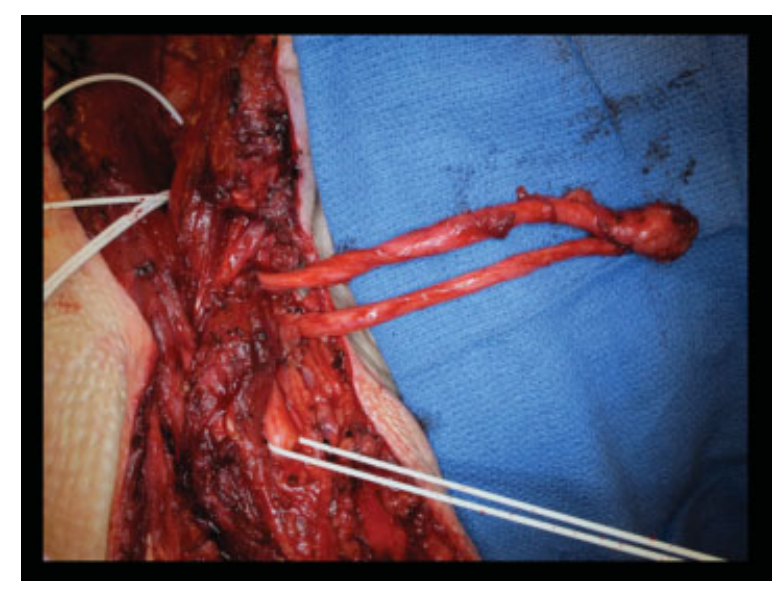

Fig. 7 Right transhumeral amputation neuromas.

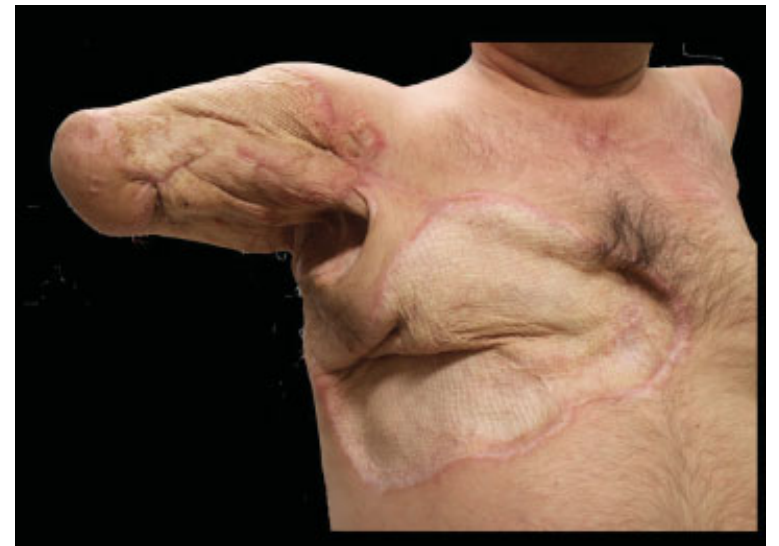

Fig. 8 Right transhumeral amputation with healed free gracilis muscle transfer with overlying skin graft.

cortically controlled muscle targets, he began rehabilitation with a new myoelectric prosthesis (described in greater detail below).

\section{Overview of Available Upper Limb Prostheses}

After upper extremity amputation, a majority of patients are fit with a prosthetic device. Multiple types of prosthetic arms are available to patients, and range from purely cosmetic to functional devices that assist in activities of daily living. Choice of prosthetic device is highly individualized, and depends on many factors such as the patient's vocation, lifestyle, and cosmetic desires.

Functional devices are classified as either body-powered or electric-powered, though hybrid devices using both approaches can also be configured. Body powered prostheses use harnesses and cables to convert patient's shoulder movement into movement of the prosthesis. Protracting the shoulder produces tension on the cable, which then moves a prosthetic hand or hook, wrist, or elbow. Such devices are robust, but rely on patient's physical strength. Electric-powered prostheses instead use motors to create movement.

Most patients with powered devices have myoelectric prostheses. Myoelectric prostheses use the EMG signals from the residual limb to control the motors. With conventional myoelectric control for patients without TMR, a single flexion and extension pair of muscles in the patient's residual limb is used to control the prosthesis. With this conventional myoelectric control, the patient uses these two muscle EMG signals to control a joint, then cocontracts the muscles to switch to another joint (-Fig. 9). ${ }^{21}$ Ideal locations are over independently controlled muscle groups, with limited extraneous EMG noise from nearby musculature-known as muscle cross talk. In patients without TMR, typical locations are over antagonistic muscle pairs. Examples include wrist flexors and extensors for a transradial amputee, biceps brachii and triceps brachii for a transhumeral amputee, and pectoralis major and infraspinatus for a shoulder disarticulation amputee. The difference in EMG amplitude between the 


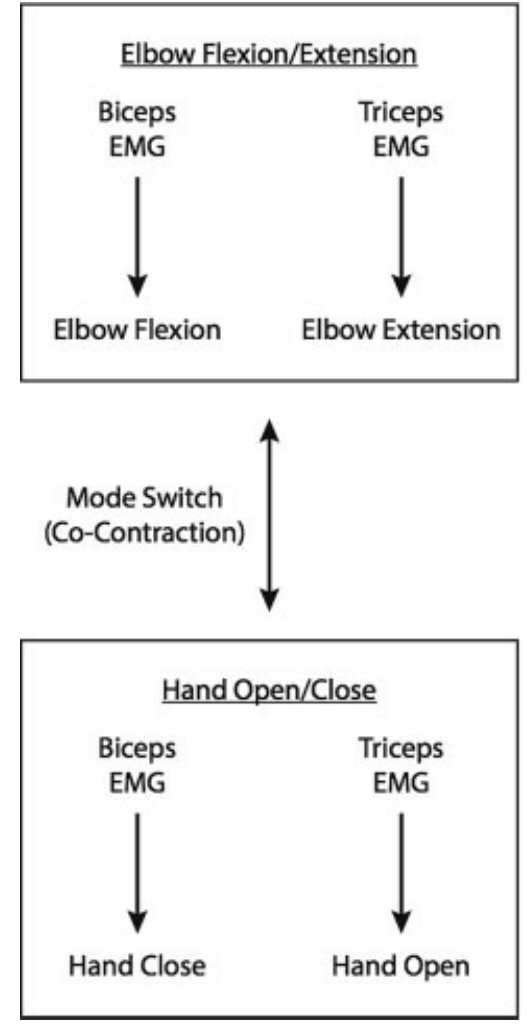

Fig. 9 Conventional myoelectric control for transhumeral amputation without targeted muscle reinnervation. Patients use an antagonistic muscle pair (biceps and triceps) to control each prosthetic degree of freedom. Therefore, for both elbow and hand mode, subjects produce an electromyographic signal (EMG) by attempting to move the lost elbow. To switch between elbow and hand modes, patients must signal a mode switch by cocontracting the biceps and triceps.

flexor site and extensor site determines the velocity of the prosthesis at a specific degree of freedom (i.e., prosthetic joint). For example, a transhumeral amputee may use a biceps contraction to close a prosthetic hand and a triceps contraction to open a prosthetic hand. Typically, only one pair of independent control sites can be located in the residual limb, thus limiting prosthesis control to one degree of freedom at a time. To switch which degree of freedom they wish to control, patients must signal a mode-switch to the prosthesis, typically by providing a quick cocontraction of both muscles in the antagonistic pair. There are very many variations of this basic control scheme, but all of these conventional myoelectric control systems are based on the amplitude of the EMG signal. A small subset of electric-powered devices uses electromechanical switches, such as force sensitive resistors, to control the motors. Switches are mounted inside the socket, and a patient pushes into the switch to activate the motor.

Conventional myoelectric control in patients without TMR becomes increasingly less intuitive with higher levels of amputation because physiologically appropriate muscle groups are less available. Whereas a transradial amputee can contract wrist flexors and extensors to sequentially control a prosthetic wrist and hand, transhumeral amputees must use proximal biceps and triceps muscles to control the elbow and all distal prosthetic functions. This conventional control is slow and cumbersome. Subjects have reported that without TMR, conventional control "[is not] very normal" and that switching between different modes was "awkward and slow."22

\section{Benefits of TMR for Prosthesis Control}

\section{Benefits of TMR with Conventional Myoelectric Control}

After TMR, there are now more than just one pair of muscles available for control of the prosthesis. Targeted muscle reinnervation surgery allows patients with above-elbow amputations to use conventional myoelectric control more intuitively by establishing new myoelectric control sites. After TMR surgery, patients typically have four myoelectric control sites: two for elbow control and two for hand control. The control of the prosthetic hand and elbow are done using physiologically appropriate nerves (-Fig. 10). This enables much more natural, intuitive, and easier operation of the prosthesis. Attempting to flex the lost elbow causes contraction of the normal heads of the biceps and triceps, allowing intuitive control of the prosthetic elbow. Attempting to close the lost hand causes the medial head of the biceps (which is reinnervated by the median nerve) to contract, thereby causing the prosthetic hand to close intuitively. Furthermore, TMR produces four control signals that can be independently modulated thereby enabling simultaneous control of an elbow and hand, instead of the traditional mode switching that is used prior to TMR.

Targeted muscle reinnervation surgery has been shown to provide improvement of prosthesis control and of function with both objective and subjective outcome measures. In a case series of five amputees, subjects consistently exhibited higher performance metrics during in-laboratory testing of functionality. ${ }^{23}$ During the box and blocks test, a validated and standardized measure of gross hand function, subjects moved $323 \pm 151 \%$ more blocks in the allotted time after TMR surgery, compared with conventional control prior to surgery. Similarly, in a clothespin relocation test, subjects were able to manipulate and relocate clothespins an average of $49 \pm 12 \%$ more quickly than they had been able to do prior to TMR surgery. Assessment of motor and process skills (AMPS) test also showed consistent improvement in scores reflecting motor skills and cognitive burden in task planning. Subjectively, patients reported an improvement in controllability as well. The Disabilities of the Arm, Shoulder, and Hand (DASH) measure, which questions subjects on how their disability has affected social activities, work, and activities of daily living, indicated lesser degree of disability after TMR surgery in a series of three subjects. In one subject's own words: “To be able to think 'open your hand' and your hand opens, rather than using your elbow, which is so unnatural-I wouldn't change it for anything." 22

\section{Benefits of TMR with Pattern Recognition Myoelectric Control}

The conventionally used myoelectric control algorithms can provide improved control after TMR, but do not take full advantage of the neural information available after surgery. 


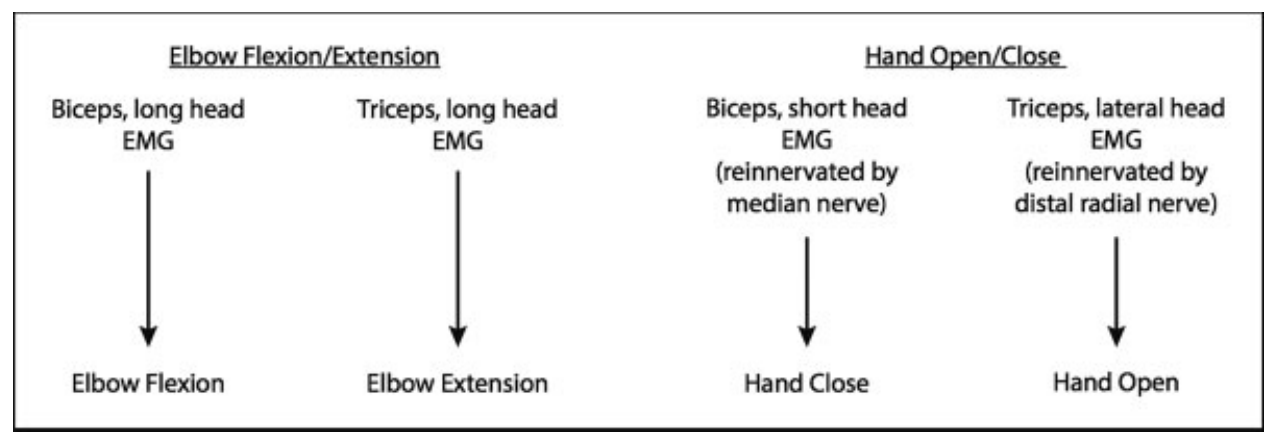

Fig. 10 Conventional myoelectric control for transhumeral amputation, with targeted muscle reinnervation. Patients use natively innervated biceps and triceps to control the elbow and reinnervated biceps and triceps to control the hand. Subjects produce an electromyographic signal (EMG) by attempting to move the corresponding joint in the lost limb. No mode switching is required.

The nerves transferred in TMR originally innervated distal muscles that actuated many joints in the intact limb. Electromyography from TMR sites therefore has potential to provide information relevant for the control of many different prosthetic degrees of freedom. For example, the reinnervation site for the median nerve, which originally innervated anterior forearm and thenar muscles, may provide useful information to direct pronation, wrist flexion, and hand grasp activity. Indeed, high-density EMG recordings of TMR sites show a multitude of neural control information present in these reinnervation sites. ${ }^{24}$ Detailed mappings of EMG amplitudes across the chest of a shoulder disarticulation patient with TMR show distinctive patterns of activity as a patient attempts a variety of thumb, finger, and wrist movements. Conventional myoelectric control methods, which only use EMG amplitude at specific myoelectric control sites, cannot take advantage of this complex information. Thus, although EMG from TMR sites have the potential to provide advanced control of wrist and grasp patterns, conventional algorithms limit the patient to simple movements of a hand and elbow.

Pattern recognition algorithms instead use machine learning approaches to predict the patient's intended movement, and take advantage of the complex neural information provided by TMR. This technology has recently become commercially available to patients. ${ }^{25}$ Using pattern recognition, patients with TMR can control elbow flexion/extension, wrist flexion/extension, wrist rotation, and multiple hand grasps, simply by attempting the corresponding movement in their amputated limb. Pattern recognition control was first demonstrated by a TMR patient with physical prostheses in $2009,{ }^{26}$ and has recently been incorporated into prostheses for home use. Scheme and Englehart provide a comprehensive discussion on the details of pattern recognition control. ${ }^{27}$ Briefly, patients provide example contractions for each of the possible prosthesis movements (e.g., elbow flexion, supination, hand open, etc.), and the algorithm learns which EMG patterns correspond to each intended movement. When a patient later wishes to move the prosthesis, the algorithm predicts the intended movement from the new pattern of EMG signals. Initially, pattern recognition control required a desktop computer and a virtual environment to train the algorithms. Recent advancements now use the prosthesis itself as a visual prompt to guide training. Training can be completed outside of the clinic in $<2$ minutes. ${ }^{28}$

In contrast to conventional control methods, pattern recognition does not use only EMG amplitudes recorded from specific antagonistic muscle pairs. Instead, pattern recognition uses numerous EMG recordings, over both natively and reinnervated muscle, to globally characterize the patient's contractions. Prosthetists do not need to specifically locate optimal myoelectric control sites, which is frequently an arduous task in the configuration of conventional myoelectric control. Instead, pattern recognition control has been shown equally effective when using a generic grid of electrodes on the residual limb to record EMG. ${ }^{29}$ Furthermore, pattern recognition uses features other than signal amplitude to characterize the EMG activity, therefore using more signal information than conventional myoelectric control. Such signal features may include the number of times the EMG signal changes direction, or how often the signal crosses the zero baseline. Numerous different EMG feature sets and machine-learning algorithms have been investigated, and many have been found to perform well for pattern recognition. ${ }^{30}$

Pattern recognition myoelectric control has been shown to provide excellent control of multiple prosthetic degrees of freedom in the laboratory. Amputee subjects with TMR demonstrated performance comparable to age-matched nonamputee control subjects when completing prosthesis-control tasks in a virtual environment. ${ }^{26}$ Furthermore, real-time testing with physical prostheses have demonstrated that use of pattern recognition allows TMR patients substantial performance benefits in the above-described box and blocks and clothespin relocation tests. ${ }^{31}$ Patients moved $40 \%$ more blocks, and completed clothespin relocation in $25 \%$ less time when using pattern recognition than when using conventional myoelectric control. The patients also subjectively preferred pattern recognition control to conventional control.

\section{Case Example (Continued)}

Prior to TMR surgery, the above-described patient used a hybrid prosthesis on his transhumeral side, which included a passive (nonmoving) elbow and a myoelectric hook. He had limited function using this device, and frequently did not 
wear it because of discomfort. After shoulder disarticulation TMR surgery on his left side, the patient was initially fit with a conventional myoelectric prosthesis. This system used four myoelectric control sites to conventionally control elbow flexion/extension and hand open/close. A force-sensitive resistor allowed the patient to switch hand open/close to instead control wrist rotation. Upon discharge from occupational therapy, he was able to pick up and release light objects, but had difficulty feeding himself or drinking from a bottle.

The patient was subsequently fit bilaterally with patternrecognition myoelectric prostheses. Using these devices, he had sequential control of elbow flexion extension, wrist rotation, and hand open/close. During functional tests such as the box and blocks test and the clothespin relocation test, the patient demonstrated similar performance between his right and left sides, despite the difference in amputation level (box and blocks test, [R] $14.3 \pm 0.3$ blocks vs. [L] $11.0 \pm 1.5$ blocks; clothespins, [R] $59.7 \pm 10.6 \mathrm{~s}$ vs. [L] $60.6 \pm 11.5$ s). ${ }^{32}$ The results suggest the intuitiveness of control with TMR and pattern recognition control, as higher-level amputation would otherwise be expected to provide poorer performance in functional tasks.

During occupational therapy, the patient demonstrated proficiency in feeding himself finger foods, eating with a fork, and drinking from a water bottle. He was also able to perform common household tasks, such as carrying a laundry basket and placing retrieving and replace items from a refrigerator. He was unable to do many of these tasks before TMR surgery or before use of pattern-recognition control. The patient reported satisfaction with his current prostheses, and frequent device use for functional tasks including yard work and drinking from bottles.

\section{Future Advances}

Targeted muscle reinnervation has provided an exciting new way to interface with a patient's nervous system to provide intuitive control of myoelectric prostheses for patients with above elbow amputations. There continues to be significant research and development to improve artificial limbs, both with TMR itself and with new technologies that will be synergistic with TMR. Potential advances include the use of TMR in transradial amputees, advanced prosthetic arm systems, and new mechanical and electrical interfaces.

Though TMR has primarily been used for individuals with above-elbow amputations, the benefits of reinnervation surgery could also be extended to transradial amputees. This is a sizeable population, as nearly $40 \%$ of all major (above wrist) amputations are at the transradial level. ${ }^{33}$ Because transradial amputation leaves extrinsic finger and wrist musculature in the residual limb, such patients without TMR are still candidates to use pattern-recognition control. Indeed, transradial amputees without TMR have similar accuracy in patternrecognition control of wrist movement as high-level amputees with TMR. However, transradial amputees cannot perform multiple hand-grasp patterns as reliably as higher-level amputees with TMR. ${ }^{26,34}$ It is hypothesized that such differences stem from the presence of reinnervated EMG repre- senting intrinsic finger muscle activity. Targeted muscle reinnervation in transradial amputees is therefore expected to extend such benefits in grasp selection, and is currently being investigated at the Rehabilitation Institute of Chicago. ${ }^{35}$

Additional work is also being pursued to improve the control algorithms of powered prostheses. In particular, much research is dedicated to providing simultaneous control of multiple prosthetic joints. Even with TMR surgery, current algorithms are limited to sequentially controlling the degrees of freedom, or at most controlling two at a time. This limitation prevents prosthetic devices from mimicking the multijoint coordination that is present in intact limbs. Current efforts have focused on extending traditional pattern-recognition algorithms, ${ }^{36}$ as well modeling neural patterns of muscle coactivations, known as muscle synergies. ${ }^{37}$

Targeted muscle reinnervation and other advances in prosthesis control have coincided with newly developed prosthetic arm systems. Targeted muscle reinnervation and pattern recognition control have allowed for more intuitive control of a greater number of prosthesis movements, which new advanced prosthetic devices are able to mechanically actuate. There has been a recent surge of multifunction prosthetic hands, including the Bebionic (Bebionic, Leeds, England) and i-LIMB (Touch Bionics, Livingston, England) hands. ${ }^{38,39}$ These devices can be configured to control multiple complex grasp patterns, allowing patients to operate the hands for a variety of activities of daily living. Currently, prosthetic wrists are limited only to rotation; however, several groups are developing two- and three-degree-offreedom wrists for commercialization. ${ }^{40-42}$ DARPA's Revolutionizing Prosthetics Program from 2005 to 2009 funded the development of two advanced prototype arms. DEKA Research and Development Corporation designed a 10-degreeof-freedom modular arm system, whose early prototypes provided excellent control for amputees with TMR. Additionally, Johns Hopkins Applied Physics Laboratory produced a 22-degree-of-freedom modular prosthetic limb system, with individually actuated fingers. However, due to its complexity and cost, it is unlikely to be made commercially available in its current form.

Finally, research efforts have also focused on improving the mechanical and electrical interfaces between the patient and the prosthesis. Currently, prostheses are suspended from the body's soft tissue. Prostheses therefore often feel uncomfortably heavy to the user. Osseointegration, where the prosthesis is percutaneously attached to the amputated bone, is a promising investigational method of suspension. One European group has successfully implanted devices in over 100 people around the world, though the system is not currently approved by the Food and Drug Administration for use in the United States. ${ }^{43,44}$ New devices are also being developed to enhance the electrical interface with the device. Electromyographic signals have traditionally been recorded from the skin surface. Surface records are an easy, noninvasive method of obtaining control signals for the prosthesis. However, electrodes tend to shift as patients don and doff the prosthesis, and changes in skin impedance from sweating can lead to changes in EMG signal quality. Researchers are therefore currently 
pursuing the development of wireless implantable recording devices. ${ }^{45,46}$ Such devices have the potential to provide a more chronic, stable recording interface between device and muscle. Intramuscular EMG also has different signal properties from surface EMG, such as decreased cross talk and access to deep muscles, which could allow for more new advancements in prosthesis control algorithms.

\section{Conclusion}

Targeted muscle reinnervation combined with existing and emerging prosthetic technology allows for intuitive control of myoelectric prostheses for amputees at multiple levels. For complex amputees, such as the patient presented in the case example, a strategic and orderly approach to care is essential, understanding that each patient will present unique challenges.

Acknowledgments
Illustrations were provided by Kyle Miller, MD.

\section{References}

1 Ziegler-Graham K, MacKenzie EJ, Ephraim PL, Travison TG, Brookmeyer R. Estimating the prevalence of limb loss in the United States: 2005 to 2050. Arch Phys Med Rehabil 2008;89(3):422-429

2 Fischer H. US Military Casualty Statistics: Operation New Dawn, Operation Iraqi Freedom, and Operation Enduring Freedom. Washington, DC: Congressional Research Service; 2010:1-9

3 Sears HH. Trends in upper-extremity prosthetics development. In: Bowker JH, Michael JW, eds. Atlas of Limb Prosthetics. St Louis, MO: Mosby; 1992:345-356

4 Souza JM, Cheesborough JE, Ko JH, Cho MS, Kuiken TA, Dumanian GA. Targeted muscle reinnervation: a novel approach to postamputation neuroma pain. Clin Orthop Relat Res 2014;472(10): 2984-2990

5 Jensen TS, Krebs B, Nielsen J, Rasmussen P. Phantom limb, phantom pain and stump pain in amputees during the first 6 months following limb amputation. Pain 1983;17(3):243-256

6 Jensen TS, Krebs B, Nielsen J, Rasmussen P. Immediate and longterm phantom limb pain in amputees: incidence, clinical characteristics and relationship to pre-amputation limb pain. Pain 1985; 21(3):267-278

7 Pierce RO Jr, Kernek CB, Ambrose TA II. The plight of the traumatic amputee. Orthopedics 1993;16(7):793-797

8 Wood VE, Mudge MK. Treatment of neuromas about a major amputation stump. J Hand Surg Am 1987;12(2):302-306

9 Kim PS, Ko J, O'Shaughnessy KK, Kuiken TA, Dumanian GA. Novel model for end-neuroma formation in the amputated rabbit forelimb. J Brachial Plex Peripher Nerve Inj 2010;5(5):6-6

10 Kim PS, Ko JH, O’Shaughnessy KK, Kuiken TA, Pohlmeyer EA, Dumanian GA. The effects of targeted muscle reinnervation on neuromas in a rabbit rectus abdominis flap model. J Hand Surg Am 2012;37(8):1609-1616

11 Ko JH, Kim PS, O'Shaughnessy KD, Ding X, Kuiken TA, Dumanian GA. A quantitative evaluation of gross versus histologic neuroma formation in a rabbit forelimb amputation model: potential implications for the operative treatment and study of neuromas. J Brachial Plex Peripher Nerve Inj 2011;6:8-8

12 Dumanian GA, Souza JM. Surgical techniques for targeted muscle reinnervation. In: Kuiken TA, Schultz Feuser AE, Barlow AK, eds. Targeted Muscle Reinnervation: A Neural Interface for Artificial Limbs. Boca Raton, FL: CRC Press; 2013:22-43
13 O'Shaughnessy KD, Dumanian GA, Lipschutz RD, Miller LA, Stubblefield K, Kuiken TA. Targeted reinnervation to improve prosthesis control in transhumeral amputees. A report of three cases. J Bone Joint Surg Am 2008;90(2):393-400

14 Marquardt E, Neff G. The angulation osteotomy of above-elbow stumps. Clin Orthop Relat Res 1974;(104):232-238

15 Dumanian GA. Targeted muscle reinnervation and upper limb amputation. In: Thorne CHM, Gurtner GC, Chung K, et al, eds. Grabb and Smith's, Plastic Surgery. 7th ed. Philadelphia, PA: Wolters Kluwer; 2013:900-907

16 Cheesborough JE, Souza JM, Dumanian GA, Bueno RA Jr. Targeted muscle reinnervation in the initial management of traumatic upper extremity amputation injury. Hand (NY) 2014;9(2):253-257

17 Lowery MM, Stoykov NS, Taflove A, Kuiken TA. A multiple-layer finite-element model of the surface EMG signal. IEEE Trans Biomed Eng 2002;49(5):446-454

18 Urist MR, Nakagawa M, Nakata N, Nogami H. Experimental myositis ossificans: cartilage and bone formation in muscle in response to a diffusible bone matrix-derived morphogen. Arch Pathol Lab Med 1978;102(6):312-316

19 Shehab D, Elgazzar AH, Collier BD. Heterotopic ossification. J Nucl Med 2002;43(3):346-353

20 Garland DE. A clinical perspective on common forms of acquired heterotopic ossification. Clin Orthop Relat Res 1991;(263):13-29

21 Williams TW. Control of powered upper extremity prostheses. In: Meier RH, Atkins DJ, eds. Functional Restoration of Adults and Children with Upper Extremity Amputation. New York, NY: Demos Medical Publishing; 2004:207-224

22 Miller LA, Stubblefield KA, Lipschutz RD, et al. Surgical and functional outcomes of targeted muscle reinnervation. In: Kuiken TA, Schultz Feuser AE, Barlow AK, eds. Targeted Muscle Reinnervation: A Neural Interface for Artificial Limbs. Boca Raton, FL: CRC Press; 2013:149-164

23 Miller LA, Stubblefield KA, Lipschutz RD, Lock BA, Kuiken TA. Improved myoelectric prosthesis control using targeted reinnervation surgery: a case series. IEEE Trans Neural Syst Rehabil Eng 2008;16(1):46-50

24 Zhou P, Lowery MM, Englehart KB, et al. Decoding a new neural machine interface for control of artificial limbs. J Neurophysiol 2007;98(5):2974-2982

25 Coapt. Homepage. Available at: http://coaptengineering.com/. Accessed September 18, 2014

26 Kuiken TA, Li G, Lock BA, et al. Targeted muscle reinnervation for real-time myoelectric control of multifunction artificial arms. JAMA 2009;301(6):619-628

27 Scheme E, Englehart K. Electromyogram pattern recognition for control of powered upper-limb prostheses: state of the art and challenges for clinical use. J Rehabil Res Dev 2011;48(6):643-659

28 Simon AM, Lock BA, Stubblefield KA. Patient training for functional use of pattern recognition-controlled prostheses. J Prosthet Orthot 2012;24(2):56-64

29 Tkach DC, Young AJ, Smith LH, Rouse EJ, Hargrove LJ. Real-time and offline performance of pattern recognition myoelectric control using a generic electrode grid with targeted muscle reinnervation patients. IEEE Trans Neural Syst Rehabil Eng 2014;22(4):727-734

30 Hargrove LJ, Englehart K, Hudgins B. A comparison of surface and intramuscular myoelectric signal classification. IEEE Trans Biomed Eng 2007;54(5):847-853

31 Hargrove LJ, Lock BA, Simon AM. Pattern recognition control outperforms conventional myoelectric control in upper limb patients with targeted muscle reinnervation. Paper presented at: The 35th Annual International Conference of the IEEE Engineering in Medicine and Biology Society; July 3-7, 2013; Osaka, Japan

32 Kuiken TA, Turner K, Soltys N, Dumanian GA. First clinical fitting of an individual after bilateral TMR with intuitive pattern recognition control. Paper presented at: Myoelectric Controls Symposium; August 18-24, 2014; Fredericton, New Brunswick, Canada 
33 Dillingham TR, Pezzin LE, MacKenzie EJ. Limb amputation and limb deficiency: epidemiology and recent trends in the United States. South Med J 2002;95(8):875-883

34 Li G, Schultz AE, Kuiken TA. Quantifying pattern recognition-based myoelectric control of multifunctional transradial prostheses. IEEE Trans Neural Syst Rehabil Eng 2010;18(2):185-192

35 Kuiken TA. Application of Targeted Reinnervation for People with Transradial Amputation (NIH R01HD081525). Chicago, IL: Rehabilitation Institute of Chicago; 2014

36 Young AJ, Smith LH, Rouse EJ, Hargrove LJ. A comparison of the real-time controllability of pattern recognition to conventional myoelectric control for discrete and simultaneous movements. J Neuroeng Rehabil 2014;11(1):5

37 Jiang N, Rehbaum H, Vujaklija I, Graimann B, Farina D. Intuitive, online, simultaneous, and proportional myoelectric control over two degrees-of-freedom in upper limb amputees. IEEE Trans Neural Syst Rehabil Eng 2014;22(3):501-510

38 Medynski C, Rattray B. Bebionic prosthetic design. Paper presented at: 2011 MyoElectric Controls/Powered Prosthetics Symposium; August 14, 2011; Fredericton, New Brunswick, Canada

39 Otr OV, Reinders-Messelink HA, Bongers RM, Bouwsema H, Van Der Sluis CK. The i-LIMB hand and the DMC plus hand compared: a case report. Prosthet Orthot Int 2010;34(2):216-220

40 Kyberd PJ, Lemaire ED, Scheme E, et al. Two-degree-of-freedom powered prosthetic wrist. J Rehabil Res Dev 2011;48(6):609-617
41 Sensinger J, Lipsey J, Sutton L, Thomas A. Development of a lightweight, low-cost, myoelectric prosthesis. Paper presented at: 39th Annual Meeting and Scientific Symposium of the American Academy of Orthotists \& Prosthetists; February 20-23, 2013; Orlando, FL

42 Sears HH, Iversen EK, Christenson J, Jacobs T. Integrated system of new electric TD and wrist components - Stage 1 developments. Paper presented at: Myoelectric Controls Symposium; August 1824, 2014; Fredericton, New Brunswick, Canada

43 Brånemark R, Brånemark PI, Rydevik B, Myers RR. Osseointegration in skeletal reconstruction and rehabilitation: a review. J Rehabil Res Dev 2001;38(2):175-181

44 Palmquist A, Jarmar T, Emanuelsson L, Brånemark R, Engqvist H, Thomsen P. Forearm bone-anchored amputation prosthesis: a case study on the osseointegration. Acta Orthop 2008;79(1): 78-85

45 Weir RF, Troyk PR, DeMichele GA, Kerns DA, Schorsch JF, Maas H. Implantable myoelectric sensors (IMESs) for intramuscular electromyogram recording. IEEE Trans Biomed Eng 2009;56(1): 159-171

46 Bercich RA, Joseph J, Gall OZ, Maeng J, Kim Y-J, Irazoqui PP. Implantable device for intramuscular myoelectric signal recording. Paper presented at: The 34th Annual International Conference of the IEEE EMBS; August 28-September 1, 2012; San Diego, CA 